\title{
Stress-induced cortisol hampers memory generalization
}

\author{
Lisa C. Dandolo and Lars Schwabe \\ Department of Cognitive Psychology, Institute of Psychology, University of Hamburg, 20146 Hamburg, Germany
}

\begin{abstract}
Integrative encoding and generalization across past experiences depends largely on the hippocampus, an area known to be particularly sensitive to stress. Yet, whether stress influences the ability to generalize memories is unknown. We exposed volunteers to a stressor or a control manipulation before they completed an acquired equivalence task probing memory generalization. While stress left learning performance intact, it reduced participants' ability to generalize and this deficit was directly linked to the cortisol response to the stressor. These findings show that stress, presumably through the action of glucocorticoids, creates rather rigid memories that are difficult to transfer to novel situations.
\end{abstract}

[Supplemental material is available for this article.]

Learning and memory can be supported by distinct systems that operate in parallel but differ in the mode of information processing (McDonald and White 1993; Voermans et al. 2004; White et al. 2013). The most prominent of these systems is based on the medial temporal lobe (MTL), including the hippocampus, and supports mainly episodic, spatial, or relational ("cognitive") memory (Scoville and Milner 1957; O'Keefe and Nadel 1978; Eichenbaum 1999; Burgess et al. 2002). This system is also essential for memory integration during encoding, associative memory, and flexible learning (Eichenbaum 2004; Shohamy and Wagner 2008; Zeithamova and Preston 2010; Horner and Burgess 2013; Schlichting et al. 2014; Backus et al. 2016; Richter et al. 2016). However, memory performance may remain intact even after MTL damage (Knowlton et al. 1996; Corkin 2002). These remaining memory functions depend on other memory systems, such as the dorsal striatum that supports habit-based learning and memory (Knowlton et al. 1996; Packard and Knowlton 2002). Compared with the MTL-dependent memory system, however, dorsal striatum-dependent memory lacks the flexibility that is required to generalize experiences to novel situations (Myers et al. 2003).

Converging lines of evidence indicate that stress induces a shift from hippocampal to dorsal striatal control of learning (Kim et al. 2001; Schwabe et al. 2007; Wingard and Packard 2008; Packard and Goodman 2012; Schwabe 2013; Schwabe and Wolf 2013). Glucocorticoids, released during stressful encounters, play a key role in this bias toward striatal learning after stress (Schwabe et al. 2010a, 2013; Vogel et al. 2015). Interestingly, the stress-induced shift in the engagement of multiple memory systems does not necessarily affect task performance (Schwabe et al. 2010a; Schwabe and Wolf 2012), raising the question how the putative alteration in the involvement of distinct memory systems changes the outcome of learning under stress. If stress biases the engagement of multiple memory systems at the expense of the MTL-based system, and this system is, as outlined above, essential for memory generalization, it could be hypothesized that stress impairs the generalization of memories. However, whether stress may indeed disrupt memory generalization is currently unknown.

We tested the impact of stress on memory generalization in 64 healthy, nonsmoking adults ( 32 women; age: mean $=25.00$ $\mathrm{yr}, \mathrm{SEM}=0.50 \mathrm{yr}$ ) without medication intake or a history of any mental or neurological disorders. All participants provided writ-

\section{Corresponding author: lars.schwabe@uni-hamburg.de}

Article is online at http://www.learnmem.org/cgi/doi/10.1101/lm.042929.116. ten informed consent, and the study protocol was approved by the local ethics committee. To control for diurnal variations of cortisol, all testing took place in the afternoon. Participants were randomly assigned to a stress or a control condition (16 men and 16 women per group). In the stress condition, participants underwent the standardized Trier Social Stress Test (TSST) (Kirschbaum et al. 1993). Briefly, participants gave a 5-min free speech promoting their candidacy for a job tailored to their interests and performed a 5-min mental arithmetic task performed in front of a rather cold and nonreinforcing panel. Participants were videotaped and could see themselves on a TV screen behind the panel. In the control condition, participants held a free speech on a topic of their choice and performed an easy mental arithmetic task; there was no panel and no video recordings were taken. To assess the effectiveness of the stress induction, we took subjective stress ratings, blood pressure measurements, and saliva samples at several time points before and after the experimental manipulation. From saliva, we analyzed cortisol concentrations using a luminescence immunoassay (IBL). We subdivided the stress group into cortisol low- and cortisol high-responders based on a median-split for the baseline to peak cortisol increase; the median increase was $3.07 \mathrm{nmol} / \mathrm{L}$, which indicates a substantial stressrelated increase (Miller et al. 2013). Based on canonical statistical criteria (Tabachnick and Fidell 2013), four participants (two controls and two cortisol low responders) were identified as outliers ( $>2$ SD below/above the mean) and thus excluded from the analyses, leaving a sample of 60 participants.

Twenty-five minutes after the onset of the stress/control manipulation, participants performed a computerized acquired equivalence paradigm that was adapted from Myers et al. (2003). Participants had to learn which fish (consequent stimulus) belonged to a specific individual (antecedent stimulus, Fig. 1). The antecedent stimuli consisted of eight pictures of persons, differing in three features: age, gender, and hair color. The consequent stimuli consisted of eight drawings of differently colored fish. In each acquisition trial, participants saw one person together with two different fish and were asked to indicate by button press which fish belonged to the shown person. Their answer was marked and they received immediate feedback about the correct

(C) 2016 Dandolo and Schwabe This article is distributed exclusively by Cold Spring Harbor Laboratory Press for the first 12 months after the full-issue publication date (see http://learnmem.cshlp.org/site/misc/terms.xhtml). After 12 months, it is available under a Creative Commons License (AttributionNonCommercial 4.0 International), as described at http://creativecommons. org/licenses/by-nc/4.0/. 

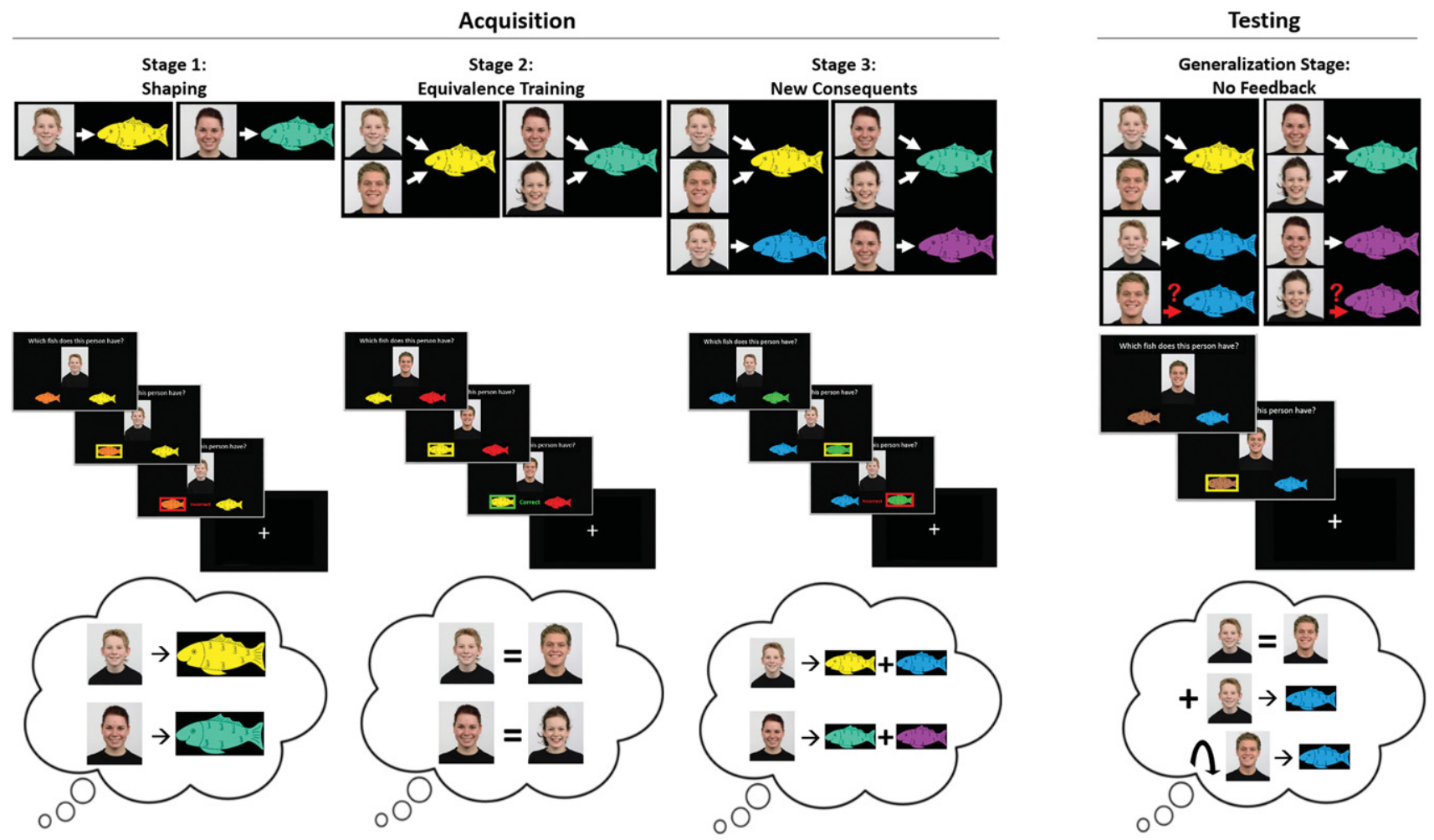

Figure 1. Acquired equivalence task. In three Acquisition Stages, participants learned stimulus relationships and equivalences: (1) in the first stage ("shaping"), participants learned four antecedent (person)-consequent (fish) stimulus pairings (only two examples shown here); (2) in the second stage ("equivalence training"), four new persons (antecedents) were introduced, with each new person owning the same fish as one of the previously introduced persons, thereby creating equivalences between two antecedent stimuli; (3) in the third stage ("new consequents"), the original four persons (antecedents) were presented with new fish (consequents), showing that a person can have two kinds of fish. On each trial, participants saw one person and two fish and were asked to indicate which fish belongs to this person. Participants received feedback about the correct answer. In a subsequent Testing Phase, in which no feedback was provided, participants had to demonstrate their memories for the learned associations in old trials and additionally had to generalize their memories to trials that had never been trained. In these new trials, the persons (antecedents) that were newly presented in the second acquisition stage were presented with two of the new fish (consequents) introduced in the third acquisition stage, thus requiring participants to make use of the equivalence learned in acquisition stage 2.

answer. The acquisition phase comprised three stages: (1) in the first stage ("shaping"), participants learned four antecedent-consequent stimulus pairings; (2) in the second stage ("equivalence training"), four new persons (antecedents) were introduced, with each new person owning the same fish as one of the previously introduced persons, thereby creating equivalences between two antecedent stimuli, which always shared two features: gender and hair color; (3) in the third stage ("new consequents"), the original four persons (antecedents) were presented with new fish (consequents), showing that a person can have two kinds of fish (Fig. 1). In each stage, 24 new trials were presented in random order with all the trials from the previous stages. The start of a new stage was not signaled to the participants. In the testing phase, the 72 old trials of the acquisition phase were randomly mixed with 24 new trials. These new trials showed the persons (antecedents) that were newly presented in the second acquisition stage presented with two of the new fish (consequents) introduced in the third acquisition stage (Fig. 1). Thus, these trials probed whether participants would show acquired equivalence, i.e., generalize the previously learned associations to trials that had not been shown during training. To rule out new learning effects, participants did not receive feedback in this testing phase. In total, participants took $\sim 16$ min to complete the task.

The subjective and physiological measures confirmed the successful stress induction by the TSST (Fig. 2; Supplemental Results).
Overall, participants performed very well in the acquisition phase with an average performance of $\sim 85 \%$. A group $\times$ acquisition stage ANOVA showed a main effect of acquisition stage $\left(F_{(1.7,99.6)}=14.72, P<0.001, \eta^{2}=0.20\right)$, with the performance constantly improving across acquisition stages. Most important, however, there was neither a group $\times$ acquisition stage interaction nor a main effect of group (both $F<0.14$, both $P>0.84$ ). Furthermore, cortisol high responders did not differ in their acquisition performance from low responders or controls (both $F<1.8$, both $P>0.15$ ) (Supplemental Fig. S1), and the individual cortisol increase was not associated with performance in the acquisition stages (all $r<0.09$, all $P>0.47$ ). Thus, stress and stress-induced cortisol did not affect acquisition performance.

From the data of the testing phase, we calculated a Generalization Score as the critical behavioral index of memory generalization. This score was calculated as percentage of correct answers in the 24 new trials minus percentage of correct answers in the 24 old trials that were introduced in acquisition stage 3 plus a constant of 100 . Thus, this parameter takes the interdependence of initial learning and subsequent memory generalization into account and allows us to disentangle participants' memory for the learned associations from their ability to generalize these memories to novel situations.

Although stress had no impact on performance in the stage 3 trials that were included in the testing phase $(P=0.99)$ (Supplemental Table S2), thus ruling out a simple retrieval deficit, 

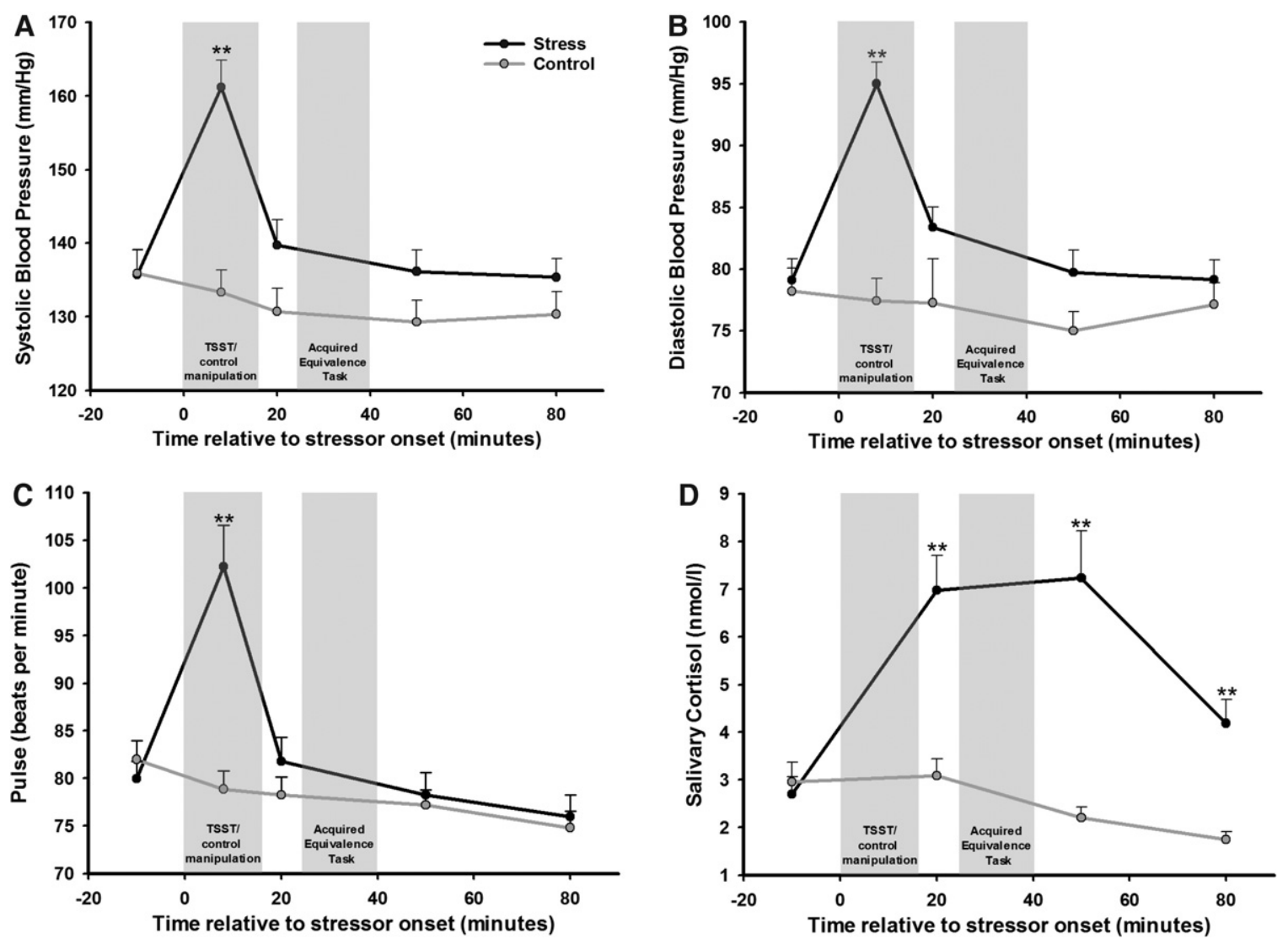

Figure 2. Physiological responses elicited by the exposure to the Trier Social Stress Test (TSST). ( $A$ ) Systolic blood pressure, (B) diastolic blood pressure, (C) pulse, and $(D)$ salivary cortisol concentrations increased in response to the TSST but not after the control manipulation. Details of the statistical analyses as well as data on the subjective stress responses are presented in the Supplemental Material. The gray bars denote the timing and duration of the treatment (TSST vs. control manipulation) and the Acquired Equivalence Task, respectively. Error bars indicate standard errors of the mean. $(* *) P<0.001$.

analysis of the Generalization Score showed that the ability to generalize was significantly decreased in the stress group relative to the control group $(t=-2.11, P=0.04$, Fig. 3A). Dividing the stress group into cortisol low- and high-responders revealed that this effect was mainly driven by stressed individuals showing a strong cortisol response (main effect of cortisol response group: $\left.F_{(2,57)}=3.10, P=0.05\right)$ : cortisol high responders showed a significantly lower Generalization Score than controls $(P<0.05)$, whereas the Generalization Score of the cortisol low responders did not significantly differ from the control group $(P>0.95$; high responder vs. low responder $P>0.60$ ) (Fig. 3B). The critical role of the stress-induced cortisol increase was also reflected in a pronounced negative correlation between the cortisol increase and the Generalization Score ( $r=-0.31, P=0.02)$ (Fig. 3C). In addition to the association with cortisol, the Generalization Score was also negatively correlated with the indicators of sympathetic nervous system activity measured during the experimental manipulation: systolic blood pressure $(r=-0.30, P=0.02)$, diastolic blood pressure $(r=-0.26, P<0.05)$, and pulse $(r=-0.34, P=0.01$; Supplemental Fig. S2). Based on a prominent model that assumes that stress effects on learning and memory require interacting cortisol and sympathetic activity (Roozendaal et al. 2006), we further calculated a product term of the $z$-transformed cortisol increase and $z$-transformed pulse and obtained a negative correlation between this interaction term and the Generalization Score $(r=-0.27, P=0.04$; Supplemental Fig. S2).

Using an "acquired equivalence" task, we show here that stress interferes with memory generalization processes. Although participants' acquisition performance remained unaffected by stress, stress impaired their ability to generalize the learned stimulus pairs to novel pairings. This stress-induced generalization deficit was mainly observed in participants who showed a strong cortisol increase to the stressor and was directly correlated with the individual cortisol response, indicating a crucial role of cortisol in the impaired memory generalization after stress.

Memory generalization based on the integration of overlapping events into a linked mnemonic representation is thought to depend critically on the hippocampus (Shohamy and Wagner 2008; Zeithamova and Preston 2010; Backus et al. 2016). In line with this idea, neuropsychological findings indicate that the generalization of memories is severely impaired in patients with hippocampal atrophy (Myers et al. 2002, 2003) or other hippocampal dysfunctions (Shohamy et al. 2010; Ivleva et al. 2012). Thus, it seems reasonable to assume that the stress-induced generalization deficit was due to an impairing effect of stress on hippocampal functioning. Indeed, there is ample evidence from neurophysiological and neuroimaging studies, suggesting that stress before learning may interfere with hippocampal activity (Kim and Diamond 2002; Schwabe and Wolf 2012). Furthermore, stress is known to favor dorsal striatal learning, at the expense of hippocampal learning (Wingard and Packard 2008; Schwabe et al. 2010c; Schwabe and Wolf 2013). Together, the proposed stress-induced impairment of hippocampal functioning and the bias towards striatal learning may have interfered with participants' capacity to encode the learned associations as discrete representations. This discrete encoding, however, is required for 

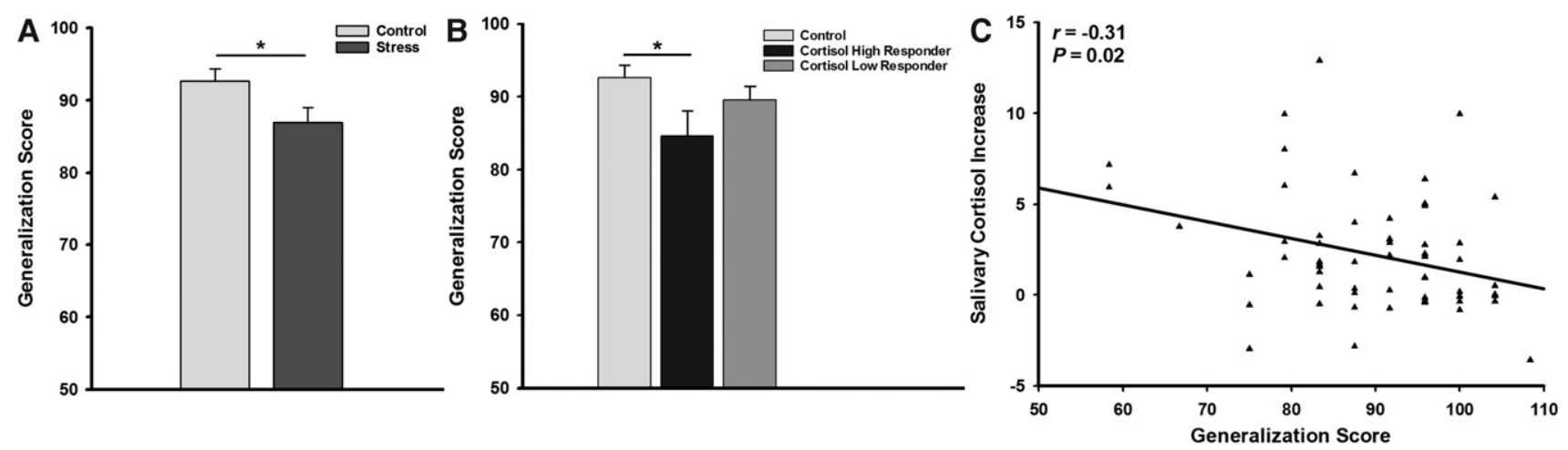

Figure 3. Impact of the stress exposure and cortisol on memory generalization. The Generalization Score reflects participants' capacity to generalize the acquired associations to new stimulus pairs in relation to their performance for initially learned associations. A Generalization Score of 100 would reflect equal performance in the old and new trials, while a lower score than 100 indicates that performance was worse in the new trials, suggesting difficulties to generalize. (A) Stress resulted in a significantly lower Generalization Score compared with the control manipulation. (B) This generalization deficit was mainly due to the impairment of stressed participants showing a strong cortisol response to the stressor ("cortisol high responders"). (C) The role of cortisol is further reflected in the negative correlation of the Generalization Score with the salivary cortisol increase from baseline to peak. Error bars indicate standard errors of the mean. $\left(^{*}\right) P<0.05$.

remembering specific details of events, to detect overlap between experiences and consequently to generalize across them.

The putative stress-induced shift from hippocampal toward dorsal striatal learning after stress could also explain why acquisition performance remained intact after stress. Specifically, a neuropsychological study that used the same acquired equivalence task showed that both patients with hippocampal atrophy and those with striatal dysfunction were able to learn this task to a certain extent, yet that patients with hippocampal atrophy were not able to generalize the learned associations to novel stimulus pairs (Myers et al. 2003). A stress-induced bias toward striatal learning may thus not necessarily impair acquisition performance but become apparent when the flexibility of memory is probed in the generalization test. This interpretation is in line with previous evidence showing that stress may alter the nature of learning without affecting task performance (Kim et al. 2001; Schwabe et al. 2007, 2010a; Seehagen et al. 2015). The changes in learning, however, become apparent when the environment changes or new information is added, revealing that memories formed under stress are rather rigid and less flexible.

Because we administered stress before learning and the testing phase followed immediately after the acquisition phase, one might argue that stress may not have necessarily altered memory encoding but rather the retrieval of this information in the testing phase, pointing to the well-known retrieval deficit after stress (de Quervain et al. 1998; Buchanan et al. 2006). However, such a retrieval deficit should be reflected in impaired performance in all trials of the testing phase, including those from the acquisition phase. Yet, we observed no impairment in these old trials, thus rendering a simple retrieval deficit unlikely. One might still argue that even if the observed effects are not due to a retrieval deficit they could be owing to impaired inference processes in the testing phase. Although this latter possibility cannot be fully ruled out, neuroimaging evidence indicates that successful generalization in an acquired equivalence task similar to the one used here is mainly mediated by hippocampal activity during encoding (Shohamy and Wagner 2008). It is thus tempting to speculate that the observed generalization deficit was mainly due to less integrative encoding of events, making it difficult to generalize across them. The explicit dissociation of stress effects on the encoding and generalization phases, however, remains a challenge for future studies.

The generalization deficit after stress was directly linked to the activity of cortisol. More specifically, the generalization deficit was only found in participants that showed a strong cortisol response to the stressor and correlated with the increase in cortisol. The critical role of cortisol in stress effects on cognition in general is well known (Joels 2001; de Quervain et al. 2003). Prefrontal and medial temporal areas are among the primary targets of cortisol in the brain (de Kloet et al. 2005; Joels and Baram 2009) and also the shift from hippocampal to dorsal striatal memory depends on cortisol, presumably acting via the MR (Schwabe et al. 2013; Vogel et al. 2015, 2016). Glucocorticoids, such as cortisol, however, do not act in isolation. In particular, it is well established that glucocorticoids may interact with noradrenergic arousal to modulate memory functions (Roozendaal et al. 2006, 2009; Schwabe et al. $2010 b)$. In line with this view, the generalization deficit obtained here was also associated with the product term of cortisol increase and autonomic activation.

In sum, our results show that stress can interfere with our ability to generalize memories to new situations and that cortisol, in interaction with autonomic arousal, is critically involved in this effect. Importantly, this generalization deficit came without impairments in acquisition performance, underlining that even when learning performance is unaffected by stress, stress may have important effects on the nature (e.g., flexibility) of memory. As the generalization across past experiences is essential for flexible decision-making and behavior, stress-induced impairments in memory generalization may have considerable consequences in many areas of our lives. In educational contexts, for instance, the ability to transfer and generalize knowledge to novel contexts is crucial. In addition, the finding that stress may disrupt our ability to generalize appropriately and, at a more general level, the flexibility of memory may aid our understanding of stress-related mental disorders such as anxiety disorders or major depression.

\section{Acknowledgments}

This work was supported by the German Research Foundation (DFG grant SCHW1357/12-1). We thank Vera Schmidt, Maria Protopopova, Jördis Hansen, and Amina Shah for their assistance during data collection.

\section{References}

Backus AR, Schoffelen JM, Szebenyi S, Hanslmayr S, Doeller CF. 2016 Hippocampal-prefrontal $\theta$ oscillations support memory integration. Curr Biol 26: 450-457. 
Buchanan TW, Tranel D, Adolphs R. 2006. Impaired memory retrieval correlates with individual differences in cortisol response but not autonomic response. Learn Mem 13: 382-387.

Burgess N, Maguire EA, O'Keefe J. 2002. The human hippocampus and spatial and episodic memory. Neuron 35: 625-641.

Corkin S. 2002. What's new with the amnesic patient H.M.? Nat Rev Neurosci 3: 153-160.

de Kloet ER, Joels M, Holsboer F. 2005. Stress and the brain: From adaptation to disease. Nat Rev Neurosci 6: 463-475.

de Quervain DJ, Roozendaal B, McGaugh JL. 1998. Stress and glucocorticoids impair retrieval of long-term spatial memory. Nature 394: 787-790.

de Quervain DJ, Henke K, Aerni A, Treyer V, McGaugh JL, Berthold T, Nitsch RM, Buck A, Roozendaal B, Hock C. 2003. Glucocorticoid-induced impairment of declarative memory retrieval is associated with reduced blood flow in the medial temporal lobe. Eur J Neurosci 17: 1296-1302.

Eichenbaum H. 1999. The hippocampus and mechanisms of declarative memory. Behav Brain Res 103: 123-133.

Eichenbaum H. 2004. Hippocampus: Cognitive processes and neural representations that underlie declarative memory. Neuron 44: $109-120$.

Horner AJ, Burgess N. 2013. The associative structure of memory for multi-element events. J Exp Psychol Gen 142: 1370-1383.

Ivleva EI, Shohamy D, Mihalakos P, Morris DW, Carmody T, Tamminga CA. 2012. Memory generalization is selectively altered in the psychosis dimension. Schizophr Res 138: 74-80.

Joels M. 2001. Corticosteroid actions in the hippocampus. J Neuroendocrinol 13: $657-669$.

Joels M, Baram TZ. 2009. The neuro-symphony of stress. Nat Rev Neurosci 10: $459-466$

Kim JJ, Diamond DM. 2002. The stressed hippocampus, synaptic plasticity and lost memories. Nat Rev Neurosci 3: 453-462.

Kim JJ, Lee HJ, Han JS, Packard MG. 2001. Amygdala is critical for stress-induced modulation of hippocampal long-term potentiation and learning. J Neurosci 21: 5222-5228.

Kirschbaum C, Pirke KM, Hellhammer DH. 1993. The 'Trier Social Stress Test' - a tool for investigating psychobiological stress responses in a laboratory setting. Neuropsychobiology 28: 76-81.

Knowlton BJ, Mangels JA, Squire LR. 1996. A neostriatal habit learning system in humans. Science 273: 1399-1402.

McDonald RJ, White NM. 1993. A triple dissociation of memory systems: Hippocampus, amygdala, and dorsal striatum. Behav Neurosci 107: $3-22$.

Miller R, Plessow F, Kirschbaum C, Stalder T. 2013. Classification criteria for distinguishing cortisol responders from nonresponders to psychosocial stress: Evaluation of salivary cortisol pulse detection in panel designs. Psychosom Med 75: 832-840.

Myers CE, Kluger A, Golomb J, Ferris S, de Leon MJ, Schnirman G, Gluck MA. 2002. Hippocampal atrophy disrupts transfer generalization in nondemented elderly. J Geriatr Psychiatry Neurol 15: 82-90.

Myers CE, Shohamy D, Gluck MA, Grossman S, Kluger A, Ferris S, Golomb J, Schnirman G, Schwartz R. 2003. Dissociating hippocampal versus basal ganglia contributions to learning and transfer. J Cogn Neurosci 15: $185-193$.

O'Keefe J, Nadel L. 1978. The hippocampus as a cognitive map. Clarendon Press, Oxford.

Packard MG, Goodman J. 2012. Emotional arousal and multiple memory systems in the mammalian brain. Front Behav Neurosci 6: 14

Packard MG, Knowlton BJ. 2002. Learning and memory functions of the basal ganglia. Аnпи Rev Neurosci 25: 563-593.

Richter FR, Chanales AJ, Kuhl BA. 2016. Predicting the integration of overlapping memories by decoding mnemonic processing states during learning. Neuroimage 124: 323-335.
Roozendaal B, Okuda S, de Quervain DJ, McGaugh JL. 2006. Glucocorticoids interact with emotion-induced noradrenergic activation in influencing different memory functions. Neuroscience 138: 901-910.

Roozendaal B, McEwen BS, Chattarji S. 2009. Stress, memory and the amygdala. Nat Rev Neurosci 10: 423-433.

Schlichting ML, Zeithamova D, Preston AR. 2014. CA1 subfield contributions to memory integration and inference. Hippocampus 24: $1248-1260$.

Schwabe L. 2013. Stress and the engagement of multiple memory systems: integration of animal and human studies. Hippocampus 23: 1035-1043. Schwabe L, Wolf OT. 2012. Stress modulates the engagement of multiple memory systems in classification learning. J Neurosci 32: 11042-11049.

Schwabe L, Wolf OT. 2013. Stress and multiple memory systems: from 'thinking' to 'doing'. Trends Cogn Sci 17: 60-68.

Schwabe L, Oitzl MS, Philippsen C, Richter S, Bohringer A, Wippich W, Schachinger H. 2007. Stress modulates the use of spatial versus stimulus-response learning strategies in humans. Learn Mem 14: $109-116$.

Schwabe L, Schachinger H, de Kloet ER, Oitzl MS. 2010a. Corticosteroids operate as a switch between memory systems. J Cogn Neurosci 22: $1362-1372$.

Schwabe L, Tegenthoff M, Hoffken O, Wolf OT. 2010b. Concurrent glucocorticoid and noradrenergic activity shifts instrumental behavior from goal-directed to habitual control. J Neurosci 30: 8190-8196.

Schwabe L, Wolf OT, Oitzl MS. 2010c. Memory formation under stress: quantity and quality. Neurosci Biobehav Rev 34: 584-591.

Schwabe L, Tegenthoff M, Hoffken O, Wolf OT. 2013. Mineralocorticoid receptor blockade prevents stress-induced modulation of multiple memory systems in the human brain. Biol Psychiatry 74: 801-808.

Scoville WB, Milner B. 1957. Loss of recent memory after bilateral hippocampal lesions. J Neurol Neurosurg Psychiatry 20: 11-21.

Seehagen S, Schneider S, Rudolph J, Ernst S, Zmyj N. 2015. Stress impairs cognitive flexibility in infants. Proc Natl Acad Sci 112: 12882-12886.

Shohamy D, Wagner AD. 2008. Integrating memories in the human brain: hippocampal-midbrain encoding of overlapping events. Neuron 60: 378-389.

Shohamy D, Mihalakos P, Chin R, Thomas B, Wagner AD, Tamminga C. 2010. Learning and generalization in schizophrenia: effects of disease and antipsychotic drug treatment. Biol Psychiatry 67: 926-932.

Tabachnick BG, Fidell LS. 2013. Using multivariate statistics. Pearson Education, Boston.

Voermans NC, Petersson KM, Daudey L, Weber B, Van Spaendonck KP, Kremer HP, Fernandez G. 2004. Interaction between the human hippocampus and the caudate nucleus during route recognition. Neuron 43: 427-435.

Vogel S, Klumpers F, Kroes MC, Oplaat KT, Krugers HJ, Oitzl MS, Joels M, Fernandez G. 2015. A stress-induced shift from trace to delay conditioning depends on the mineralocorticoid receptor. Biol Psychiatry 78: 830-839.

Vogel S, Fernandez G, Joels M, Schwabe L. 2016. Cognitive adaptation under stress: A case for the mineralocorticoid receptor. Trends Cogn Sci 20: $192-203$.

White NM, Packard MG, McDonald RJ. 2013. Dissociation of memory systems: The story unfolds. Behav Neurosci 127: 813-834.

Wingard JC, Packard MG. 2008. The amygdala and emotional modulation of competition between cognitive and habit memory. Behav Brain Res 193: $126-131$.

Zeithamova D, Preston AR. 2010. Flexible memories: Differential roles for medial temporal lobe and prefrontal cortex in cross-episode binding. $J$ Neurosci 30: 14676-14684.

Received May 13, 2016; accepted in revised form August 17, 2016. 


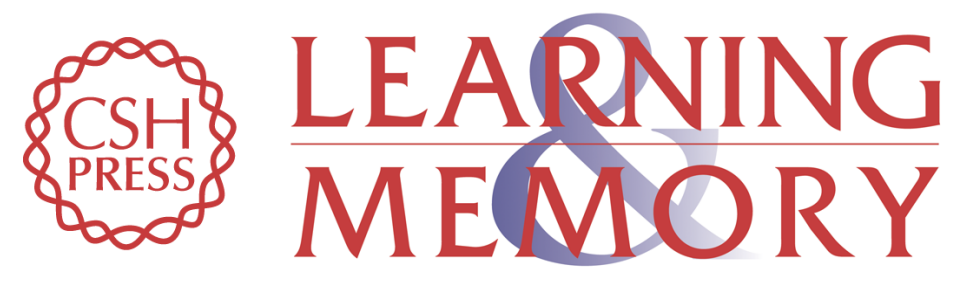

\section{Stress-induced cortisol hampers memory generalization}

Lisa C. Dandolo and Lars Schwabe

Learn. Mem. 2016, 23:

Access the most recent version at doi:10.1101/lm.042929.116

Supplemental http://learnmem.cshlp.org/content/suppl/2016/11/09/23.12.679.DC1

References This article cites 45 articles, 10 of which can be accessed free at: http://learnmem.cshlp.org/content/23/12/679. full.html\#ref-list-1

Creative This article is distributed exclusively by Cold Spring Harbor Laboratory Press for the Commons License first 12 months after the full-issue publication date (see http://learnmem.cshlp.org/site/misc/terms.xhtml). After 12 months, it is available under a Creative Commons License (Attribution-NonCommercial 4.0 International), as described at http://creativecommons.org/licenses/by-nc/4.0/.

Email Alerting Receive free email alerts when new articles cite this article - sign up in the box at the Service top right corner of the article or click here. 